\title{
ANTROPOLOGIA EDUCACIONAL: A CONSOLIDAÇÃO DE UMA SUBÁREA EPISTEMOLÓGICA
}

\author{
EDUCATIONAL ANTHROPOLOGY: \\ THE CONSOLIDATION OF AN \\ EPISTEMOLOGICAL SUB-AREA
}

\author{
Adelcio Machado dos Santos \\ adelciomachado@gmail.com \\ Doutor em Engenharia e Gestão do Conhecimento Universidade Federal de Santa Catarina (UFSC). Docente \\ e Pesquisador da Universidade Alto Vale do Rio do Peixe (UNIARP).

\section{Rodrigo Regert} \\ regert.rodrigo@gmail.com \\ Mestre em Desenvolvimento e Sociedade (UNIARP). Mestre em Educação pela Universidad Tecnológica \\ Intercontinental (UTIC). Docente da UNIARP.
}

\section{RESUMO}

O objeto da Antropologia engloba as maneiras físicas primitivas e atuais relacionadas ao homem e as suas manifestações culturais. Atribui-se a essa área epistêmica a faina de proceder às generalizações, estabelecendo princípios explicativos do desenvolvimento e da formação das sociedades e culturas humanas. A Antropologia Cultural constitui a subárea mais ampla, visto que abrange o estudo do humano em sua ontologia, ou seja, gerador de cultura. Igualmente, analisa as culturas humanas no tempo e no espaço, suas origens e desenvolvimento, suas semelhanças e discrepâncias. Tem seu interesse voltado para o conhecimento do comportamento cultural humano, obtido por aprendizado, considerando-o em todas as suas dimensões. Tal como para a Antropologia e para a Educação, a diversidade humana representa grande riqueza de problema heurístico a demandar pesquisa. Ademais, a abordagem antropológica da educação opera com missão relevante: analisa a construção do conhecimento contextualizado socialmente. Em epítome, a Antropologia Educacional se configura jovem, contudo auspiciosa subárea epistemológica. Por isso o objetivo do presente artigo é analisar a contribuição que a Antropologia pode oferecer a Educação. O mesmo trata-se de uma pesquisa descritiva com base bibliográfica. Conclui-seque aAntropologia é plural e está sempre aberta ao diferente, não permitindo que o seu saber se consolide de forma precipitada. A Antropologia da Educação viabiliza uma análise adequada da complexidade, da interculturalidade e das novas tecnologias que são as grandes incógnitas do saber pedagógico.

Palavras-chave: Antropologia. Educação. Cultura.

\section{ABSTRACT}

The object of Anthropology encompasses primitive and current physical ways related to humans and their cultural manifestations. The work of making generalizations is attributed to this epistemic area, establishing explanatory principles for development and formation of human societies and cultures. Cultural Anthropology is the broadest sub-area, since that covers the study of the humans in their ontology, i.e. generating culture. Likewise, it examines the human cultures in time and space, 
their origins and development, their similarities and discrepancies. The approach has interest focused on the knowledge of human cultural behavior, obtained through learning, considering it in all its dimensions. As for the Anthropology and Education, human diversity is a wealth of problem demanding heuristic search. And besides, the anthropological approach operates with the relevant mission of education: analyses the construction of knowledge socially contextualized. In epitome, Educational Anthropology takes shape of a young, however, auspicious epistemological sub-area. So the purpose of this article analyzes the contribution that anthropology can offer to education. It is a descriptive research based on bibliography. It is concluded that Anthropology is plural and is always open to the different, not allowing its knowledge to be suddenly consolidated. Anthropology of Education enables an appropriate analysis of complexity, interculturality and new Technologies that are great unknowns of pedagogical knowledge.

Keywords: Anthropology. Education. Culture.

\section{INTRODUÇÃO}

Considerando que a educação,além de ser uma ciência,sempre constitui uma atividade intencional a ser medida pela referência a uma finalidade ou projeto, ela configura práticas sociais movidas por interesses inerentes à cultura, felicidade dos indivíduos e sobrevivência da espécie humana.

Contudo os novos conceitos e estruturas, gestados pela Antropologia da Educação, nascem e permanecem provisórios e tensos, embora estejam sempre em busca de consistência argumentativa e analítica, de modo a oferecer potencial explicativo e estruturante para a elaboração de teorias e práticas pedagógicas.

Ademais, essa subárea epistemológica constitui domínio primordial do saber pedagógico atual, caracterizado pelo pluralismo e pela diversidade. Nenhuma teoria pedagógica produz sozinha o saber necessário à educação. Por conseguinte, a abordagem antropológica da Educação pode prestar relevante contributo para o avanço científico.

Com isso, o presente artigo tem o objetivo de analisar a contribuição que a Antropologia pode oferecer a Educação e para que isso seja atingido o mesmo foi pensado de três formas: inicialmente será descrito o sentido etimológico que a Antropologia tem; em seguida, o conceito antropológico de cultura; e pôr fim será mencionado a relação existente entre Antropologia e Educação. Para isso, o estudo deu-se de através de uma pesquisa descritiva com base bibliográfica.

\section{ANTROPOLOGIA}

Etimologicamente, o termo Antropologia significa o estudo do humano. Em outras palavras é a ciência da humanidade, ela se preocupa em avaliar cientificamente o ser humano na sua totalidade, o que lhe confere, segundo Marconi e Presotto (1987), um tríplice aspecto:

1. Ciência Social - propõe conhecer o ser humano como elemento complementar de grupos organizados.

2. Ciência Humana - volta-se designadamente para o humano como um todo: suas crenças, sua história, usos e costumes, filosofia, linguagem, entre outros.

3. Ciência Natural - interessa-se pelo conhecimento psicossomático do humano e seu desenvolvimento. 
Já Hoebel e Frost (apud MARCONI; PRESOTTO, 1987) definem a Antropologia como a ciência da humanidade e da cultura que possui relação com as artes e busca sentir e comunicar a maneira de viver de povos específicos, é também uma disciplina humanística.

Destarte, a Antropologia tem uma dimensão biológica, como Antropologia física; uma dimensão sociocultural, como Antropologia Social e/ou Antropologia Cultural; e uma grandeza filosófica, como Antropologia Filosófica. Sem dúvida, a tarefa que se atribui à Antropologia é muito vasta, o que facilita a proliferação de subdivisões e paradigmas distintos agrupados sob esta denominação comum. (BARRIO, 2005).

Todavia a diversidade dos seus campos de interesse, constitui-se em uma ciência polarizadora, que precisa da colaboração de outras áreas do saber, mas conserva sua unidade, uma vez que seu enfoque de interesse é a cultura humana.

A Antropologia tende ao conhecimento completo do humano, o que torna suas perspectivas muito mais abrangentes.

Deste modo, uma conceituação mais vasta a define como a ciência que estuda o homem, suas produções e seu comportamento. O seu interesse está no ser humano como um todo - ser cultural e ser biológico e -, preocupando-se em revelar os fatos da natureza e da cultura. Arrisca compreender a existência humana em todos os seus aspectos, no tempo e no espaço, partindo do princípio da estrutura biopsíquica. Procura também a compreensão das manifestações culturais, do comportamento e da vida social.

O objeto da Antropologia engloba as maneiras físicas primitivas e atuais do homem e suas manifestações culturais. Atribui-se ao antropólogo a tarefa de proceder as generalizações, estabelecendo princípios explicativos do desenvolvimento e da formação das sociedades e culturas humanas.

Hoebel e Frost (apud MARCONI; PRESOTTO, 1987) afirmam que a Antropologia fixa como seu objetivo o estudo da humanidade como um todo e nenhuma outra ciência analisa metodicamente todas as manifestações do ser humano e da atividade humana de maneira tão integrada.

Assegurar que a Antropologia consiste na ciência do humano não quer dizer muito, pois qualquer disciplina entre ciências humanas trata do homem e é, portanto, uma ciência do humano. Trata-se, pois, de tautologia.

A definição abrangedora da Antropologia como o estudo do humano e de suas obras justifica-se, porque centra sua atenção nesta criatura, quer seja amplo ou estreito o foco de seu interesse, tendo assim uma nova ciência.

A Antropologia como ciência preocupa-se com a questão das diferenças e busca propor formas de intervenção sobre a realidade, papel a que se dirige qualquer conhecimento produzido a partir das relações entre os homens e o mundo social criado por eles. (GUSMÃO, 2008, p. 54).

A grande amplitude da ciência impõe à Antropologia o desenvolvimento de técnicas e objetivos especiais para dar unidade a seus propósitos e métodos.

A Ciência Antropológica dividiu-se em dois grandes campos: um refere-se à forma física do homem; o outro, ao seu comportamento aprendido. Chamam-se respectivamente Antropologia Física e Cultural, sendo que aquela é, em essência, biologia humana. Os antropólogos físicos estudam problemas tais como a natureza das diferenças raciais com transmissão de traços somáti- 
cos de uma a outra geração; o crescimento, desenvolvimento e decrepitude do homem. (HERSKOVITS, 1963).

Assim, os antropólogos culturais estudam, por outro lado, os processos ideados pelo humano para enfrentar o meio natural e o ambiente social; e ainda como se aprendem, conservam e transmitem de uma a outra geração corpos de costumes.

\section{CULTURA: UM CONCEITO ANTROPOLÓGICO}

A cultura, para os antropólogos em geral, constitui-se no conceito básico e central de sua ciência. O termo "cultura" não se restringe ao campo da Antropologia.

Várias áreas do saber humano valem-se dele, embora seja outra a conotação. Muitas vezes, a palavra cultura é empregada para indicar o desenvolvimento do indivíduo por meio da educação, da instrução. Nesse caso, uma pessoa culta seria aquela que adquiriu domínio no campo intelectual ou artístico.

Seria inculto o que não obteve instrução. Porém, os antropólogos não empregam os termos culto ou inculto, de uso popular, e nem fazem juízo de valor sobre esta ou aquela cultura, pois não consideram uma superior a outra.

Elas apenas são diferentes em nível de tecnologia ou integração de seus elementos. Para os antropólogos, a cultura tem definição ampla. Engloba as maneiras comuns e aprendidas da vida, transmitidos pelos indivíduos e grupos, em sociedade. (MARCONI; PRESOTTO, 1987).

A cultura, portanto, pode ser analisada, ao mesmo tempo, sob vários enfoques: ideias, crenças, normas, atitudes, padrões de conduta, abstração do comportamento, instituições, técnicas e artefatos.

A Antropologia cultural é o campo mais amplo da ciência antropológica. Abrange o estudo do homem como ser cultural, ou seja, fazedor de cultura.

Pesquisa as culturas humanas no tempo e no espaço, suas origens e desenvolvimento, suas semelhanças e diferenças. Tem enfoque de interesse voltado para o conhecimento do comportamento cultural humano, obtido por aprendizado, considerando-o em todas as suas dimensões. O humano dimana do meio cultural em que se socializou.

Ele é um herdeiro de um extenso processo acumulativo, que reflete o conhecimento e a experiência adquiridos pelas abundantes gerações que o antecederam. A manipulação apropriada e criativa desse patrimônio cultural admite as inovações e as invenções.

Para Barrio (2005) a Antropologia cultural é o estudo e descrição dos comportamentos aprendidos que caracterizam os diferentes grupos humanos. O antropólogo cultural tem que se ocupar das obras materiais e sociais que o homem criou através de sua história e que lhe permitiram fazer frente a seu meio ambiente e relacionar-se com seus congêneres.

À semelhança do verificado em toda a Ciência Humana, seu desígnio básico consiste no problema da relação entre modos de comportamento instintivo e adquirido, bem como o das bases biológicas gerais que servem de estrutura às aptidões culturais. 
Assim, no campo cultural, o antropólogo desenvolve recursos e técnicas de pesquisa, ambos ligados à observação de campo. Este é o seu laboratório, onde aplica a técnica da observação direta, que se completa com a entrevista e a utilização de formulários para registro de dados.

A análise da cultura de uma formação social estabelece uma reconstituição da realidade, que é organizada a partir da consciência que dela têm os portadores da cultura. Sem se fixar, obviamente, aos aspectos conscientes da conduta, é por meio deles, em sua relação com o comportamento evidente, que a cultura pode ser reconstituída. (DURHAM, 2004). Importa reconhecer que o conceito foi construído em função de problemas específicos da investigação antropológica, os quais dizem respeito ao estudo dos povos chamados primitivos, isto é, sociedades relativamente indiferenciadas.

De certo modo, é possível dizer que os aspectos gerais do conceito de cultura podem ser apreendidos como um conjunto de pressupostos que decorrem do modo pelo qual a Antropologia concebeu seu objeto e definiu os problemas básicos do trabalho de campo.

Os estudiosos da cultura interessam-se, pois, por compreender como uma certa maneira de conseguir um fim determinado pode variar amplamente de um a outro povo, servindo, entretanto, a cada um para conseguir seu ajustamento diante da vida.

Tratam de especificar como as formas estabelecidas de tradição mudam no decorrer do tempo, quer em consequência de desenvolvimentos internos ou de contatos com modos estranhos, e como um indivíduo nascido numa dada sociedade absorve, usa e influencia os costumes que constituem sua herança cultural.

A Antropologia Cultural tem um campo de interesse muito mais amplo que as disciplinas afins no campo das Ciências Sociais e das Humanidades, cada uma das quais apenas se ocupa de um segmento da atividade humana. (HERSKOVITS, 1963). Além disso, a Antropologia Cultural tem com outras disciplinas afinidades mais amplas que qualquer outro ramo da Ciência matriz.

Uma vez que trata das obras do humano em toda sua grande variedade, tropeçou, até mesmo para fixar sua nomenclatura, com maiores dificuldades que qualquer outro ramo da disciplina.

Embora a mudança cultural seja ubíqua e sua análise, portanto, fundamental no estudo da vida dos grupos humanos, não se deve esquecer que, tal como qualquer aspecto do estudo da cultura, se dá em termos de ambiente e subjetividade, e não em termos em si absolutos. A cultura é ao mesmo tempo estável e mutável.

A mudança cultural pode ser estudada apenas como uma parte do problema da estabilidade cultural, a qual só pode ser entendida quando se mede a mudança relacionada com o conservantismo. Embora a cultura possa ser automática e as sanções dadas por supostas, qualquer forma aceita de ação ou de crença, qualquer instituição dentro de uma cultura tem sentido.

De acordo com o magistério de Laraia (1996), os antropólogos estão completamente convencidos de que as diferenças genéticas não são ocasionadas pelas diferenças culturais. Não existe ligação significativa entre a distribuição dos comportamentos culturais e a distribuição dos caracteres genéticos.

Assim sendo, a posição da moderna Antropologia é que a cultura age seletivamente, e não casualmente, com relação a seu meio ambiente, explorando 
certas possibilidades e limites ao desenvolvimento, para o qual as forças determinantes estão na própria cultura e na história da cultura.

Pode-se dizer que a comunicação é um processo cultural. Uma das tarefas da Antropologia moderna tem sido a reconstrução da definição de cultura, fragmentada por numerosas reformulações.

Mais explicitamente, a linguagem humana é um produto da cultura, mas não existiria se o homem não tivesse a possibilidade de desenvolver um sistema articulado de comunicação oral.

Os antropólogos sabem de fato o que é cultura, mas discordam no modo de exteriorizar este conhecimento. (LARAIA, 1996). A herança cultural, desenvolvida através de inúmeras gerações, sempre condicionou a reagir depreciativamente em relação ao comportamento daqueles que atuam fora dos padrões aceitos pela maioria da comunidade.

Assim, o fato de que o homem vê o mundo através de sua cultura tem como consequência a capacidade em considerar o seu modo de vida como o mais natural e o mais correto. Além disso, cada cultura ordena a seu modo o mundo que a circunscreve e que esta ordenação dá um sentido cultural à aparente agitação das coisas naturais.

A Antropologia Cultural, ciência de caráter hermenêutico, corre o risco de dissolver o momento da diferença, do não-idêntico na emergência de um conceito universal de compreensão e de uma metodologia positiva universal de apropriação hermenêutica do estrangeiro e de cortar sob medida. (WULF, 2005).

A cultura é, sobretudo, considerada como alguma coisa nacional, e enquanto tal, ela é associada a um território preciso e a uma língua, tradições, memórias, símbolos e rituais comuns, sendo, portanto, bastante dinâmica socialmente falando.

Considerando que a dinâmica social se projeta no futuro para o ordenamento da sociedade em decorrência dos conflitos inerentes às sociedades no presente (ou seja, a futura ordem responde e depende dos conflitos presentes), o propósito da dinâmica social é revelar o movimento com base no aspecto conflituoso entre as forças que compõem o social, o que difere radicalmente de uma abordagem da dinâmica social como movimento da história em que a sociedade meramente se reproduz em relação às forças que a direcionam a um futuro de ordem social dependente da atuação destas e ainda desconhecido.(COLLINS, 2013, p. 40-41).

Cada sistema cultural está sempre em transformação. Entender esta dinâmica é importante para diminuir o choque entre as gerações e evitar comportamentos preconceituosos.

Da mesma maneira que é fundamental para a humanidade a concepção das diferenças entre povos de culturas diferentes, é preciso saber entender as diferenças que ocorrem dentro do mesmo sistema. Todavia, grande parte dos padrões culturais de um dado sistema foi copiada de outros sistemas culturais. A esses empréstimos culturais a Antropologia denomina difusão. Os antropólogos estão convencidos de que, sem a difusão, não seria possível o grande desenvolvimento atual da humanidade. 


\section{ANTROPOLOGIA DA EDUCAÇÃO}

Segundo Wulf (2005) o saber antropológico tem um papel importante tanto no campo das ciências da educação, quanto no domínio da pedagogia prática.

Para Nunes (2016) a Antropologia, ao dialogar com a Educação, tem se desenvolvido e se especializado em trabalhos que abordam questões da diversidade cultural especialmente na escola sendo relativos a práticas hegemônicas.

Deste modo, surge uma nova dimensão do saber pedagógico chamada Antropologia pedagógica ou, dito de forma mais precisa, Antropologia histórica e cultural da educação. Essa designação abre o cenário de um dos mais inovadores e desafiantes debates da educação na atualidade: a superação dos sistemas fixos e fechados de princípios e valores por uma perspectiva histórica e cultural e, portanto, sempre provisória e relativa da pedagogia.

De acordo com Gusmão (2010) a Antropologia é uma ciência do fazer-fazendo, consolidando-se pela crítica constante de seus próprios passos, ou seja, é uma ciência que ao mesmo tempo que aprende, ensina, e da mesma forma quando ensina, aprende.

Durante os anos de 1960 e 1970, aAntropologia Educacional se interessava somente pela criança vendo nela apenas um homoeducandus. Ela destacava generalidades sobre a educação da criança, sua vocação, abstraindo suas condições histórico-culturais. As diferenças culturais e históricas existentes entre as crianças, tal qual já se analisava na Antropologia Cultural, na região anglo-saxônica, pareciam menos interessantes. (WULF, 2005).

Somente no início dos anos 90, ao se descobrir a dupla historicidade e o domínio da Antropologia histórica, pôde se efetuar uma mudança de tática. Nos anos 90, foram feitas várias tentativas de se utilizar esta nova descoberta no sentido de fazer avançar a Antropologia histórico-pedagógica. Foi nesse período que fora necessário uma revisão do conhecimento científico que foi produzido pela modernidade na busca por formas alternativas de conhecimento tendo sido designado como uma crise dos paradigmas existentes. (GUSMÃO, 2008).

Dessa forma conseguiu-se enfim compreender o alcance da Antropologia comparada, que consiste na articulação de uma perspectiva geral com uma perspectiva particular.

Conforme a concepção humboldtiana, de acordo com Von Humboldt, a Antropologia comparada tem sua particularidade no fato de que trata de uma matéria empírica de modo especulativo, de um fato histórico de modo filosófico e da natureza humana no sentido de seu desenvolvimento possível. (WULF, 2005).

A Antropologia não é, portanto, mais nem unicamente empírica, nem unicamente filosófica. Trata-se, pelo contrário de conciliar o filosófico e o empírico, de compreender um fato histórico dentro de uma perspectiva filosófica, a fim de descobrir uma evolução possível da natureza humana.

A Antropologia filosófica considera que o homem é o único ser que interroga a si mesmo, sendo desse modo sujeito e objeto de sua própria interrogação. (ANDRADE, 2016). Por isso, ao estabelecer a relação entre a filosofia e a empiria, entre o transcendental e o histórico, Von Humboldtse envolve num estudo histórico-antropológico orientado pela filosofia.

Na medida em que este estudo tem como tarefa evidenciar as possibilidades de desenvolvimento, os objetivos da Antropologia juntam-se aos da 
educação. Nessa ótica, eles não visam estabelecer uma norma, mas explorar as diferenças entre cultura, períodos históricos e indivíduos. A Antropologia busca expor as particularidades da característica moral do homem comparando diferentes tipos de ser humano.

O conhecimento da característica moral parece ser o objetivo principal da Antropologia. A tarefa da Antropologia era explorar as diferenças culturais entre os indivíduos e a sociedade. Ainda que a diferença entre os tipos humanos seja muito importante, é também indispensável reconhecer que os indivíduos e as sociedades formam um conjunto.

Por conseguinte, tal Ciência visa, por um lado, explorar as diferenças entre sociedades, culturas e indivíduos; por outro, trata de compreender o ideal de humanidade em toda a diversidade e contingência.

Sendo dado que a pesquisa antropológica se encarrega de descobrir as características de diferentes sociedades, de diferentes indivíduos e agrupamentos humanos, é necessário definir a noção de característica.

A Antropologia Comparada tende a reconstruir o caráter do ser humano a partir de suas expressões e de suas maneiras. Trata-se de compreender seus traços individuais, a relação de forças que o movem e sua força interior e perfeição, muito mais que suas atitudes e fins exteriores. Ao analisar o indivíduo, é necessário distinguir o que é essencial do que é acessório, do compreender no tempo e na história, bem como sua abertura para o futuro.

Finalmente, é necessário sintetizar a diversidade dos fenômenos na unidade mais elevada. Assim, a Antropologia tem por tarefa analisar a abrangência da diversidade dos homens sem para isso desprezar a identidade.

O conhecimento do humano e sua educação estão estreitamente ligados. Educar o ser humano é impossível sem conhecê-lo, sem que estudos antropológicos sejam efetuados. Por seu lado, a Antropologia visa à educação do indivíduo e da espécie humana. A relação entre a Antropologia e a teoria da educação é contingente, há diversas possibilidades para determinar o nexo entre as duas e para realizar uma de suas possibilidades, depois de se fazer uma escolha. Se a relação entre Antropologia e educação é considerada como contingente, ela é então aberta e variável, exigindo minuciosa investigação e posicionamento em cada nova situação. Essa abertura de possibilidades exige uma escolha, pois ela deve ser delimitada em cada situação histórica específica.

O saber antropológico é adquirido através da investigação de diferentes culturas, diferentes grupos ou indivíduos. Ele ajuda a compreender especificamente cada cultura, cada grupo e cada pessoa.

De acordo com Wulf (2005), o esforço para compreender globalmente o homem, avançando-se para além do individual e incluindo as diferenças e as contingências, torna-se um desafio para a Antropologia histórica e cultural. A educação visa ligar condições exteriores da sociedade com condições interiores do indivíduo.

Para que tais processos tenham sucesso, é necessário que o indivíduo seja livre e que a sociedade crie possibilidades suficientes de educação. Somente destarte poder-se-ia produzir uma cultura complexa.

Os resultados desses processos de educação são abertos frente ao futuro. Isso quer dizer que a educação supõe o aspecto desconhecido e incerto do futuro, assim como o caráter infinito da formação humana. Os estudos de Von Humboldt sobre a linguagem unificam sua concepção de Antropologia e sua teoria da educação, acrescentando também a isso novas dimensões. (WULF, 2005). 
Destarte, tal como para a Antropologia e a teoria da educação, a diversidade histórico-empírica das línguas é para Von Humboldt uma riqueza do mundo e do homem. Entretanto tal diversidade não impede a comunicação entre os homens.

Da mesma forma que, em Antropologia, a investigação de diferentes culturas aumenta o saber sobre o ser humano, a exploração de diferenças entre as línguas faz aumentar o saber sobre o fenômeno língua.

É a linguagem que faz com que o mundo seja humano; ela traduz o mundo em um mundo do homem. Suas fronteiras são os limites da cultura e do indivíduo. Perante a importância do papel da economia do trabalho e da economia do tempo no empenho de se criar o homem universal, a questão antropológica é retomada de uma aceleração do tempo e diminuição do trabalho. O sentido e os objetos percebidos se entrecruzam não somente quando se vê, mas também quando se toca, quando se escuta e se degusta. $\mathrm{O}$ homem não vê sem pressupostos.

Por um lado, o indivíduo começa a perceber o mundo de maneira antropomórfica, isto é, partem de suas bases fisiológicas. Por outro lado, a percepção se orienta segundo dados histórico-antropológicos e culturais.

Conforme Wulf (2005) a educação exige o trabalho das representações interiores. Trabalho em discurso, mas em procura do seu conteúdo genuíno.

A educação deve, portanto, tornar os seres humanos capazes de melhor gerir essas tensões e conflitos, a fim de construir um futuro comum para a humanidade. É necessário considerar a educação como um valor em si e como um processo que dura toda a vida. Mesmo que a educação deva enfrentar as novas exigências da sociedade, da economia e da política, é necessário evitar reduzi-la a algo totalmente amarrado a essas exigências.

A educação e a formação devem tornar-se flexíveis e levar em conta a diversidade e a heterogeneidade do mundo e de suas regiões. Trata-se de ensinar aos seres humanos a viver em conjunto de forma pacífica e construtiva.

Para isso a Antropologia Pedagógica, oferece um importante suporte, que é a possibilidade de auxiliar na busca pelo conhecimento do outro, pois saber quem ele é, se torna essencial para entender e problematizar como se deu esta construção do "outro" e por conseguinte aprender a viver em conjunto. (OLIVEIRA, 2013).

Ao se ensinar a viver em conjunto, espera-se desenvolver o conhecimento dos outros e a criatividade. Entre as formas do conhecimento, deve se dar muita importância às ciências, porque elas contribuem muito para o desenvolvimento social. A educação e a formação devem, sobretudo, estar atentas ao desenvolvimento da memória, da reflexão, da imaginação, da saúde, das faculdades estéticas e comunicativas e também às necessidades concretas dos indivíduos.

O saber antropológico da educação funciona numa dupla contextualização histórica e cultural. Por um lado, para aquele que produz o conhecimento, por outro, para aquele que, nas pesquisas, se apoia nesse conhecimento, produzido num determinado contexto. Essa dupla historicidade e culturalidade torna relativo o conteúdo do saber antropológico. Ademais, a Antropologia Educacional torna-se uma Antropologia histórica e cultural da educação, que leva em conta a historicidade e a culturalidade do pesquisador e de seu objeto.

A Antropologia histórica e cultural da pedagogia procura ainda relacionar suas perspectivas e métodos com as perspectivas e métodos de seu 
objeto. Para Wulf (2005) a Antropologia da Educação tem por tarefa a análise, a organização, a reavaliação e a produção do saber através das ciências da educação, bem como a desconstrução dos conceitos da educação, numa perspectiva antropológica.

Por fim, pode-se dizer que a Antropologia da Educação inclui também a reflexão tanto sobre as competências quanto sobre os limites de seu próprio saber.

Ela analisa as dificuldades da autodefinição do homem e de sua educação, que surgem com o desaparecimento dos pontos de referências universais. A mesma mostra como as consequências dessas dificuldades dependem da relação com suas próprias condições de produção.

\section{CONSIDERAÇÕES FINAIS}

A Antropologia tem por finalidade mostrar a diversidade dos seres humanos para melhor compreender quem são os homens.

Contudo, compreender não é simplificar e negar as contradições, nesse ínterim, a Antropologia tenta compreender o homem em toda a sua complexidade. Essa é a razão pela qual ela se interessa, sobretudo, pela diferença entre as culturas, os períodos históricos, os grupos e os indivíduos.

A linguagem, a educação e a Antropologia estão estreitamente ligadas, ser humano quer dizer ser capaz de falar e de se formar. É menos importante constatar que o indivíduo é capaz de falar e de ser formado do que definir a característica de uma língua num contexto histórico específico e a cultura que ela envolve.

Ademais, é importante fazer uma análise da configuração antropológica do pensamento que a educação e a linguagem fazem, ou seja, uma crítica à imagem antropológico-humanista predominante nas reflexões sobre a educação, possibilitando uma forma diferenciada de pensar a relação pedagógica entre os sujeitos e as práticas educativas como uma experiência e um acontecimento possibilitado por meio da linguagem. (LOPES, 2011).

Investigar isso é a tarefa da Linguística e das Ciências Educacionais. Pode-se dizer que a tarefa da educação sempre foi forjada em debates, pelos quais foram sendo formadas na história das culturas humanas, imagens do "dever-ser" do homem quanto ao seu presente ou futuro, com base na memória de algum passado de ser retirado do esquecimento.

Destarte, o debate da educação precisa, principalmente, ser prático no sentido de refletir, seja crítica ou propositivamente, sobre demandas e compromissos, os quais podem mobilizar educadores e instituições, na decisão, planejamento e execução de políticas de educação.

Os educadores precisam ter uma atitude antropológica para fundamentar seu trabalho. Embora tenham um certo nível de consciência desse saber, os educadores não dispõem de mecanismos sistemáticos e rigorosos para tornar tal saber, veladamente presente em seu quotidiano, mais explícito.

É esse fundamento que a Antropologia da Educação se dispõe a desenvolver.

A Antropologia Educacional vem ao encontro da nova perspectiva de investigação que abandona sua pretensão tradicional de investigar o homem e 
o educando como seres universais em favor de uma visão menos transcendentalista e mais realista, mais datada e historicamente contextualizada.

Não se pode imaginar que a Antropologia alimente pretensões de um novo universalismo epistêmico. Ao contrário, ela preserva sempre um caráter crítico-reflexivo que, aliás, decorre necessariamente da supressão dos referenciais estáveis e universais que davam sustentação à teoria tradicional.

Antropologia é plural e está sempre aberta ao diferente, não permitindo que o seu saber se consolide de forma precipitada. A Antropologia da Educação viabiliza uma análise adequada da complexidade, da interculturalidade e das novas tecnologias que são as grandes incógnitas do saber pedagógico.

Ademais, a Antropologia da Educação se apresenta como um projeto de reflexão sistemática a respeito de alguns fenômenos inerentes à cultura moderna e contemporânea que já vêm sendo percebidos e analisados de forma isolada, pontual e desconexa por autores e teorias de diversas procedências.

Cada pesquisador, cada educador, cada professor possui um saber antropológico sem o qual ele não poderia trabalhar. Deste modo, é necessário, tanto aos que lidam com as ciências da educação quanto aos educadores, tomar consciência dos postulados antropológicos que fundamentam o trabalho educacional.

\section{REFERÊNCIAS}

ANDRADE, Paulo Raphael Oliveira. A Antropologia Filosófica de Henrique Claúdio de Lima Vaz como superação do reducionismo antropológico. 2016. 163 f. Dissertação (Programa de Estudos de Pós-Graduação em Filosofia) Curso de Mestrado em Filosofia. PUC, Pontifícia Universidade de São Paulo, São Paulo, 2016.

BARRIO, Angel B. Espina. Manual de Antropologia cultural. Recife: Massangana, 2005.

COLLINS, Victor Rodrigues. Antropologia Social e Antropologia Dinâmica.2013. 110 f. Dissertação (Programa de Estudos de Pós-Graduação) Curso de Mestrado em Ciências Sociais. PUC, Pontifícia Universidade de São Paulo, São Paulo, 2013.

DURHAM, Eunice Ribeiro. A dinâmica da cultura:ensaios de Antropologia. São Paulo: CosacNaify, 2004.

GUSMÃO, Neusa Maria Mendes de. Antropologia, Estudos Culturais e Educação:

desafios da modernidade. Pro-Posições, v. 19, n. 3 (57) - set./dez. 2008. Disponível em: <http://www.scielo.br/pdf/pp/v19n3/v19n3a04>. Acesso em: 17 nov. 2017.

. PorumaAntropologia da Educação no Brasil. Pro-Posições, Campinas, v. 21, n. 2 (62), p. 259-265, maio/ago. 2010. Disponívelem: <http://www.scielo. br/pdf/pp/v21n2/v21n2a17.pdf>. Acesso em: 17 nov. 2017.

HERSKOVITS, Melville J. Antropologia cultural:man and his works.São Paulo: Mestre Jou, 1963. Tomo I.

LARAIA, Roque de Barros. Cultura:um conceito antropológico. 11. ed. Rio de Janeiro: Jorge Zahar, 1996.

LOPES, Rodrigo Barbosa. Imagem do Pensamento: do antropológico ao acontecimento na educação. 2011. 218 f. Tese (Programa de Pós-Graduação em 
Educação) - Curso de Doutorado em Educação. Universidade Estadual Paulista. UEP. Faculdade de Filosofia e Ciências, 2011.

MARCONI, Marina de Andrade; PRESOTTO, Zelia Maria Neves. Antropologia: uma introdução. 2. ed. São Paulo: Atlas, 1987.

NUNES, Virgínia de Santana Cordolino. Antropologia, diversidade sexual e educação: uma experiência etnográfica no ensino público da Bahia. 2016. 214 f. Dissertação (Programa de Pós-Graduação em Antropologia Social) - Curso de Mestrado em Antropologia Social. UFSC, Universidade Federal de Santa Catarina, Florianópolis, 2016.

OLIVEIRA, Amurabi de. O lugar da Antropologia na formação docente: um olhar a partir das Escolas Normais. Pro-Posições, v. 24, n. 2 (71), p. 27-40 maio/ago. 2013. Disponível em: $<$ https://periodicos.sbu.unicamp.br/ojs/index. php/proposic/article/view/8642532>. Acesso em: 17 nov. 2017.

WULF, Christoph. Antropologia da educação.Campinas, SP: Alínea, 2005. 